\title{
Hybrid scheme for modeling local field potentials from point-neuron networks
}

\author{
Espen Hagen ${ }^{1,2^{*}}$, David Dahmen ${ }^{1}$, Maria Stavrinou², Henrik Lindén ${ }^{3,4}$, Tom Tetzlaff', Sacha van Albada', \\ Sonja Grün ${ }^{1,5}$, Markus Diesmann ${ }^{1,6,7}$, Gaute T Einevoll ${ }^{2,8}$ \\ From 24th Annual Computational Neuroscience Meeting: CNS*2015 \\ Prague, Czech Republic. 18-23 July 2015
}

Measurement of the local field potential (LFP) has become routine for assessment of neuronal activity in neuroscientific and clinical applications, but its interpretation remains nontrivial. Understanding the LFP requires accounting for both anatomical and electrophysiological features of neurons near the recording electrode as well as the entire large-scale neuronal circuitry generating synaptic input to these cells. The direct simulation of LFPs in biophysically detailed network models is computationally daunting. Here, we instead propose a hybrid modeling scheme combining the efficiency of simplified point-neuron network models (Fig. 1A) with the biophysical principles underlying LFP generation by multicompartment neurons [1] (Fig 1C). We apply this scheme to a model representing a fullscale cortical network under about 1 square millimeter surface of cat primary visual cortex [2] (Fig. 1A,B) with layer-specific connectivity [3] to predict laminar LFPs
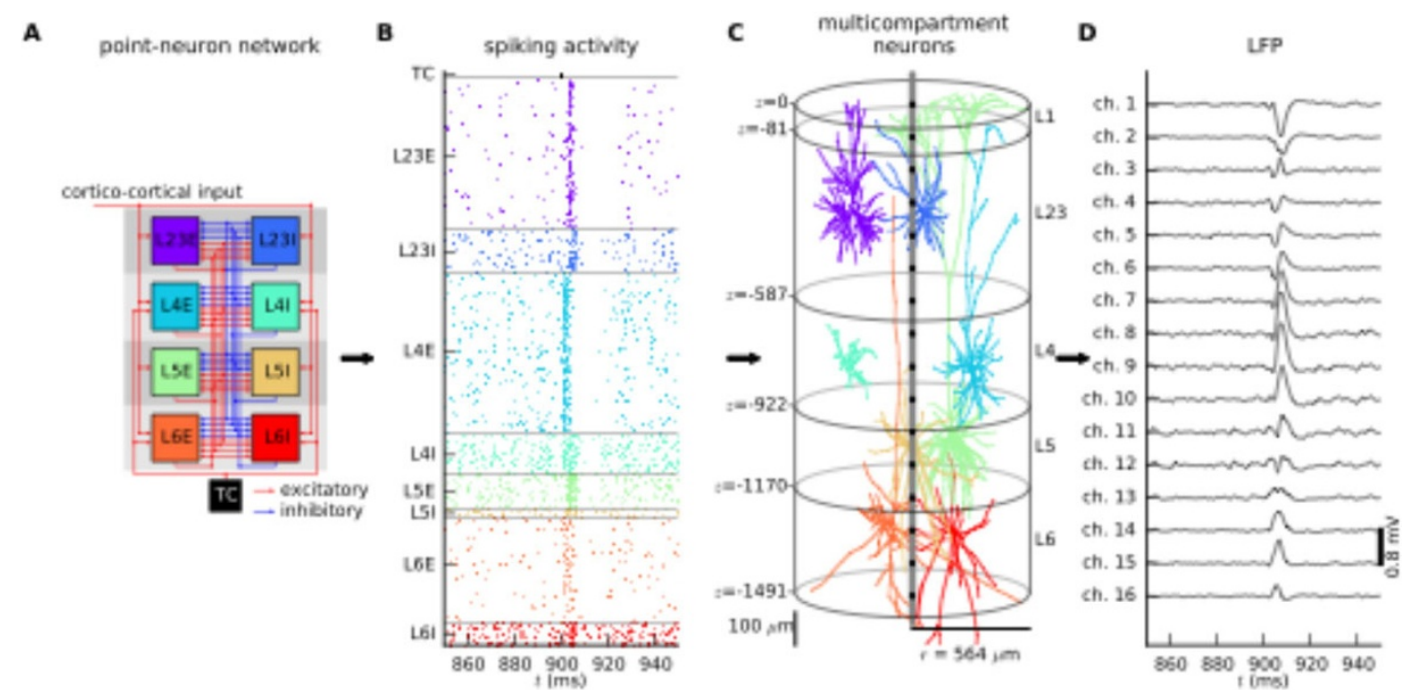

Figure 1 Overview of the hybrid scheme for modeling LFP generated by a cortical network model. A Sketch of point-neuron network model [1]. B Spikes of point neurons in the network for spontaneous and evoked activity. C Populations of multi-compartment neurons acting as LFP signal generators. D Depth-resolved LFP predicted using the hybrid scheme.

\footnotetext{
* Correspondence: e.hagen@fz-juelich.de

${ }^{1}$ Inst. of Neuroscience and Medicine (INM-6) and Inst. for Advanced

Simulation (IAS-6), Jülich Research Center and JARA, Jülich, 52425, Germany

Full list of author information is available at the end of the article
}

(c) 2015 Hagen et al. This is an Open Access article distributed under the terms of the Creative Commons Attribution License (http:// creativecommons.org/licenses/by/4.0), which permits unrestricted use, distribution, and reproduction in any medium, provided the original work is properly cited. The Creative Commons Public Domain Dedication waiver (http://creativecommons.org/publicdomain/ zero/1.0/) applies to the data made available in this article, unless otherwise stated. 
(Fig. 1D) for different network states, assess the relative contribution of local neuron populations to the LFP, investigate the role of input correlations and neuron density, and validate linear LFP predictions based on population firing rates. The hybrid scheme is accompanied by our open-source software, hybridLFPy (github. com/espenhgn/hybridLFPy).

\section{Acknowledgements}

This research was partially funded by EU Grant 604102 (HBP), EU Grant 269921 (BrainScaleS), the Helmholtz portfolio theme SMHB, the Juelich Aachen Research Alliance (JARA), and the Research Council of Norway (NFR, through ISP, NOTUR -NN4661K).

\section{Authors' details}

${ }^{1}$ Inst. of Neuroscience and Medicine (INM-6) and Inst. for Advanced

Simulation (IAS-6), Jülich Research Center and JARA, Jülich, 52425, Germany. ${ }^{2}$ Dept. of Mathematical Sciences and Technology, Norwegian University of Life Sciences, Aas, 1432, Norway. ${ }^{3}$ Dept. of Neuroscience and Pharmacology, University of Copenhagen, Copenhagen, 2200, Denmark. ${ }^{4}$ Dept. of

Computational Biology, Royal Institute of Technology (KTH), Stockholm, 10044, Sweden. ${ }^{5}$ Dept. of Biology, Theoretical Systems Neurobiology, RWTH Aachen University, Aachen, 52074, Germany. ${ }^{6}$ Dept. of Psychiatry, Psychotherapy and Psychosomatics, Medical Faculty, RWTH Aachen University, Aachen, 52074, Germany. 'Dept. of Physics, Faculty 1, RWTH Aachen University, Aachen, 52074, Germany. ${ }^{8}$ Dept. of Physics, University of Oslo, Oslo, 0316, Norway.

Published: 18 December 2015

\section{References}

1. Lindén $\mathrm{H}$, Tetzlaff T, Potjans TC, Pettersen KH, Gruen S, Diesmann M, Einevoll GT: Modeling the spatial reach of the LFP. Neuron 2011, 72:859-872.

2. Potjans TC, Diesmann M: The Cell-Type Specific Cortical Microcircuit: Relating Structure and Activity in a Full-Scale Spiking Network Model. Cerebral Cortex 2014, 24(3):785-806.

3. Binzegger T, Douglas RJ, Martin KA: A quantitative map of the circuit of cat primary visual cortex. J Neurosci 2004, 24(39):8441-8453.

doi:10.1186/1471-2202-16-S1-P67

Cite this article as: Hagen et al:: Hybrid scheme for modeling local field potentials from point-neuron networks. BMC Neuroscience 2015 16(Suppl 1):P67.

\section{Submit your next manuscript to BioMed Central and take full advantage of:}

- Convenient online submission

- Thorough peer review

- No space constraints or color figure charges

- Immediate publication on acceptance

- Inclusion in PubMed, CAS, Scopus and Google Scholar

- Research which is freely available for redistribution

Submit your manuscript at www.biomedcentral.com/submit
C Biomed Central 